\title{
EINIGE NEUE CUNONIACEEN AUS NEUGUINEA ${ }^{1}$
}

\author{
JoHANNES MATTFELD
}

\section{Ceratopetalum tetrapterum, spec. nov.}

Arbor gracilis ad $35 \mathrm{~m}$. alta, cortice crasso resinoso; rami novelli inflorescentia terminati, teretes, glabri, griseo-fusci, internodiis sat longis, stipularum cicatricibus transversis amplis. Folia opposita tetrasticha, glabra, trifoliolata, petiolata; petioli $2-4 \mathrm{~cm}$. longi, $2 \mathrm{~mm}$. crassi, apice articulati; foliola petiolulata, lateralia terminali saepe minora et brevius petiolulata vel etiam aequalia, petiolulis 5-20 mm. vel paullum ultra longis, laminae obovato-oblongae, tertia parte superiore latissimae, apice deinde subito acuminatae, basi longius breviusve cuneatim angustatae, in petiolulum decurrentes, margine crenulato-serratae, supra laeves flavovirentes, subtus costa media prominente, venis venulisque prominulis dense reticulatae, $10-18 \mathrm{~cm}$. longae, $3.5-6 \mathrm{~cm}$. latae. Inflorescentia paniculata terminalis, pluriflora, ca. $18 \mathrm{~cm}$. longa, $12 \mathrm{~cm}$. diam., in statu fructificationis defoliata; pedunculi ulteriores et pedicelli articulati pilis albis densiuscule hirtuli; pedicelli supra articulationem 3-5 mm. longi. Flores (florentes non suppetunt) tetrameri: sepala 4, petala (an desunt an delapsa?); filamenta subulata $1.5 \mathrm{~mm}$. longa 8 ; carpella 2 connata. Fructus nucamentaceus, sepalis 4 auctis, coriaceis, purpureis, lanceolatospathulatis $9.5-10 \mathrm{~mm}$. longis, $4 \mathrm{~mm}$. latis, subacutis, pilosulis vel glabrescentibus coronatus, 7-8 mm. longus, monospermus, hypanthio hemisphaerico ca. $5 \mathrm{~mm}$. alto, $6 \mathrm{~mm}$. diametiente, disperse hirtulo demum glabrescente, parte libera conoidea densius hirtula, stylis grici ibus glabris $1 \mathrm{~mm}$. longis demum caducis; pericarpii stratum exterius herbaceum demum siccum, interius coriaceo-osseum; semen unicum loculum saepius plane complens, globosum mucronatum, 5-6 mm. altum, $4 \mathrm{~mm}$. diam., apice interdum irregulariter depressum, hilo lato basilari vel oblique laterali-basilari; testa brunnea firme chartacea; albumen carnosum; embryo flavo-virens; radicula $1 \mathrm{~mm}$. longa; cotyledones transverse trapezoideae, ca. $3 \mathrm{~mm}$. longae, $4.5 \mathrm{~mm}$. latae, basi minutiuscule cordatae, planae vel saepe irregulariter et vario modo et gradu plicatae.

Südost-Neuguinea: Fly River, Oroville Camp (30 Meilen über D'Albertis Junction); häufig im Regenwald; schlanker Baum bis 35 m. hoch; Stamm mit Brettwurzeln, Rinde dick, schuppig, mit reichlichem

'Botanical Results of the Richard Archbold Expeditions. 
aromatischem Harz; Fuchtkelche purpurn (L. J. Brass nr. 7405, August 1936; Typus der Art).

Die Gattung Ceratopetalum ist meines Wissens noch nicht aus Neuguinea angegeben worden. Die bisher bekannten 4 Arten sind im westlichen Australien (Neusüdwales und Queensland) beheimatet. Von diesen haben $C$. gummiferum $\mathrm{Sm}$. (gedreite Blätter mit kleinen sitzenden Blättchen), C. apetalum Don (Blättchen einzeln, sitzend, grösser) und C. Virchowii F. v. M. (Blätter gedreit, Blättchen gestielt, gross, fast ganzrandig) fünfzählige Blüten. Aber C. succirubrum C. T. White in Proc. Roy. Soc. Queensland, 47: 60. 1936 hat mit der hier beschriebenen Art zwei wichtige Merkmale gemeinsam: vierzählige Blüten und gedreite Blätter mit gestielten Blättchen. Verschieden ist aber die Form der Blättchen; sie sind bei $C$. succirubrum kleiner $(5-12 \mathrm{~cm}$. lang, $1.5-4 \mathrm{~cm}$. breit), lanzettlich, spitz bis stumpf (nicht zugespitzt und verkehrteiförmig-länglich wie bei $C$. Virchowii und $C$. tetrapterum), und die Behaarung der Blütenstandachsen ist feiner und dichter (etwas filzig). In der Form der Blättchen ähnelt unsere Art mehr der C. Virchowii (vgl. die Abbildung in F. M. Bailey, Compr. Catalogue Queensland Plants, 173, fig. 139. 1909), in Blütendiagramm dagegen stimmt sie mit $C$. succirubrum überein. Ein Vergleich der Blütenteile ist leider nicht möglich, da von $C$. succirubrum nur Blüten beschrieben sind, während $C$. tetrapterum nur in Frucht bekannt ist.

Schizomeria katastega, spec. nov.

Arbor coma silvae humilior. Rami ulteriores inflorescentia terminati, graciles, teretes, glabri, internodiis $2-3.5 \mathrm{~cm}$. longis. Folia opposita, simplicia, petiolata, glabra; petioli $1.5-3 \mathrm{~cm}$. longi; laminae pergamenae, late oblongae vel saepius supra medium latiores et deinde obovatooblongae usque obovato-ellipticae, ca. 10-18 cm. longae, 4-9 cm. latae, apice acutae, basi cuneatim angustatae, in petiolum angustissime decurrentes, glabrae, margine distanter crenato-serratae, supra obscure virides subtus pallidiores, penninerviae, costa media subtus prominente; nervi laterales utrinque saepe ca. 11 , inter se $(1)-1.5(-2) \mathrm{cm}$. distantes; venae venulaeque subtus tenuiter prominulae, reticulatae. Inflorescentia terminalis, paniculata, parva, inter folia occulta, vix $10 \mathrm{~cm}$. longa, $8 \mathrm{~cm}$. diam.; rami infimi ex axillis foliorum supremorum, sequentes florendi tempore defoliati, ultimi bracteolis minutis suffulti; pedunculi angulati, glabri sed ultimi et pedicelli pilis paucis parvis albis disperse obsiti; pedicelli articulati, breves. Flores stellati 5-6 mm. diam., hermaphroditi, 5-meri; calycis sub toro connati segmenta ovata, acuta vel obtusiuscula, 2-2.2 mm. longa, 1.8-2 mm. basi lata, trinervia, glabra, persistentia; 
petala alba, trifurcata, 1-1.2-1.5 mm. longa, ex medio basim versus unguiculatim angustata, demum caduca; stamina 10; filamenta subulata 1.5-1.8 mm. longa; antherae ovatae, mucronatae, $0.8 \mathrm{~mm}$. longae; discus crassus pulviniformis, apice $1 \mathrm{~mm}$. latus, impresse punctatus, ante stamina episepala profunde partitus, ante stamina epipetala crenatus (gibberes deinde vero 10, anne potius 5 crenati ??), ovarii basim cingens; ovarium globosum, 1-1.5 mm. altum, $1.5 \mathrm{~mm}$. diam., glabrum vel hinc inde pilo albo stricto obsitum, 2-(rarius incomplete 3-) merum, biloculare, ovulis in quoque lovulo 4-5; styli ex basi vel apice tantum recurvi, $1 \mathrm{~mm}$. longi. Drupa ellipsoidea, obtusa, (vivo ex nota collectoris brunnea, $\pm 2 \mathrm{~cm}$. longa) sicco $15 \mathrm{~mm}$. longa, $10 \mathrm{~mm}$. lata, basi umbilicata, umbilici $3 \mathrm{~mm}$. diametientis margine sepalis persistentibus coronato, monosperma, putaminata; semen ovoideo-cylindricum, $4 \mathrm{~mm}$. longum, $2.2 \mathrm{~mm}$. diam., brunneum; embryo rectus, planus; radicula conoidea $0.8 \mathrm{~mm}$. longa; cotyledones ovato-oblongae, $2 \mathrm{~mm}$. longae, 1.2 $\mathrm{mm}$. latae.

Südost-Neuguinea: Mittelerer Fly River, Daviumbu-See, häufiger Baum der unteren Waldetage im trockeneren Regenwalde (L. J. Brass nr. 7898; September 1936 blühend; Typus der Art).- Unterer Fly River (Ostufer), Gaima, häufiger Baum der unteren Waldetage am Rande des Regenwaldes. (L. J. Brass nr. 8294; November 1936, fruchtend).

Schizomeria tegens, spec. nov.

Priori valde affinis, sed diversa foliorum forma et textura, floribus maioribus, druparum forma.- Arbor alta comam silvae formans. Folia coriacea, oblonga vel ovato-oblonga, acuta, glabra, lamina $8-14 \mathrm{~cm}$. longa, $3-5.5 \mathrm{~cm}$. lata. Inflorescentia terminalis paniculata, $15 \mathrm{~cm}$. longa, $8 \mathrm{~cm}$. diam., folia superans, pedunculi ulteriores puberuli. Calycis 3.8-4 mm. alti segmenta ovata, 2.5-2.8 mm. longa; petala ambitu obovata, 2-2.2 mm. longa, $1.2 \mathrm{~mm}$. lata, brevius trifurcata, infra medium unguiculatim contracta; stamina 10, filamenta subulata $2 \mathrm{~mm}$. longa, antherae ovatae mucronatae $0.5 \mathrm{~mm}$. longae; discus crassus, $0.8-1 \mathrm{~mm}$. altus, gibberes 5 bilobi; ovarium semiinferum, saepius 3-(rarius 2-) merum, $1 \mathrm{~mm}$. diam., $1.5 \mathrm{~mm}$. (pars libera tantum $0.5 \mathrm{~mm}$.) altum; styli $1.5 \mathrm{~mm}$. longi, rami liberi $0.5 \mathrm{~mm}$. longi recurvi; ovula in quoque loculo 4-5 (ovarium triloculare cum 15 ovulis). Drupae juveniles obovoideae (10 mm. longae putamine nunc iam nullo).

Südost-Neuguinea: Fly River, Oroville Camp; grosser Baum des Regenwaldes, das Dach des Waldes mitbildend, Rinde dick, hellbraun, korkig, rissig, Blüten weiss. (L. J. Brass nr. 7429, im August 1936 
blühend; Typus der Art).- Mittelerer Fly River, Daviumbu-See; 20 m. hoher Baum im Regenwalddach; Stamm an der Basis kantig, Rinde hart, dünn, längsrissig (L. J. Brass nr. 7497, im August 1936 mit noch nicht ganz reifen Früchten).

Diese beiden Arten stehen sich sehr nahe, unterscheiden sich aber durch die angegebenen Merkmale der Form und Textur der Blätter, der Grösse der Blüten und der Form der Früchte. Schizomeria floribunda Schlechter hat erheblich reichere Blütenstände und vor allen Dingen an der Basis abgerundete (nicht keilig verschmälerte Blätter); Sch. serrata Hochr. hat noch grössere und lang verschmälerte Blätter, die übrigen Arten haben kleinere Blätter.

Schizomeria Brassii, spec. nov.

Arbor. Rami juveniles angulati fusci, vetustiores teretes cortice griseo tecti. Folia opposita, petiolata; stipulae connatae interpetiolares, ovato-triangulae, acutae, coriaceae, cito caducae, 4-5 $\mathrm{mm}$. longae; petioli 5-15 mm. longi; laminae coriaceae, glabrae, obovato-oblongae vel obovato-ellipticae, $7-14 \mathrm{~cm}$. longae, 3-7 cm. latae, basi cuneatae in petiolum decurrentes, apice rotundatae et deinde subito brevissims acuminatae, margine crenato-serratae, penninerviae, costa media subtus prominente, nervis lateralibus sat densis, venis venulisque tenuiter prominulis reticulatae. Inflorescentia paniculata, foliis supremis brevior, fructus tantum paucos maturans. Flores (non suppetunt). Drupae globosae, 10-12 mm. longae et diametientes, e carpellis 3 constructae, umbilico basali $5-7 \mathrm{~mm}$. diametiente margine sepalis coriaceis ovatotriangulis acutis coronato.

Südost-Neuguinea (Papua): Jaritari; 3-400 m. ü. M., an Flussufern überhängend in Wäldern des Hügellandes; Baum mit hellgrauer, etwas schuppiger Rinde (L. J. Brass nr. 713, 29 November 1925 fruchtend; Typus der Art).

Diese Art ist durch kugelige Früchte ausgezeichnet. Schizomeria Pulleana O. C. Schmidt hat ellipsoidische Früchte; Sch. floribunda Schlechter, von der Früchte bisher nicht bekannt sind, hat an der Basis abgerundete Blätter.

\section{Schizomeria Whitei, spec. nov.}

Arbor mediocris ad $15 \mathrm{~m}$. alta; rami juveniles angulati, fusci, sparse pilosi mox glabrescentes, vetustiores teretes. Folia pro genere maxima, opposita, petiolata, glabra; stipulae cito caducae; petioli $8-20 \mathrm{~mm}$. longi, crassi ; laminae obscure virides, supra paullum nitentes, coriaceae, amplae, late ellipticae, $10-20 \mathrm{~cm}$. longae, $6-10 \mathrm{~cm}$. latae, apice rotundato- 
obtusissimae et leniter sinuatae, basi rotundatae vel truncatae vel etiam paullum cordatae, et saepe subito brevissime cuneatim in petiolum contractae, margine crenulis distantibus inconspicuis subintegrae, penninerviae, nervis lateralibus sat distantibus sub angulo fere recto e costa subtus prominente abeuntibus, venis venulisque subtus paullum prominulo-reticulantibus. Inflorescentiae terminalis paniculatae sat ramosae pedunculi pedicellique tomentelli, ulteriores bracteis parvis late ovatis membranaceis suffulti. Flores pentameri, rarius tetrameri; calycis extus pilosi et demum glabrescentis segmenta ex ovato oblonga vel triangula, valvata, crassa, $2.8-3 \mathrm{~mm}$. longa, $1.8-2 \mathrm{~mm}$. lata, subacuta; petala alba, trifurcata, lobis lateralibus longioribus tenuioribus, 2.8-3 mm. longa, basi ca. $0.7 \mathrm{~mm}$., media ca. 1-1.2 mm., apice 1.5-1-8 $\mathrm{mm}$. lata; stamina 10, filamenta applanato-subulata, $2-2.5 \mathrm{~mm}$. longa, antherae ovatae, mucronatae, $1 \mathrm{~mm}$. longae; disci ovarium cingentis gibberes 10 ad basim liberi, $1 \mathrm{~mm}$. longi, 0.5-0.8 mm. crassi; ovarium $0.8-1 \mathrm{~mm}$. altum, pilis longis, rectis, fuscis dense villosum; styli $2 \mathrm{~mm}$. longi, rami 2-3 ad medium connati. Drupae late ovoideae, sicco $1.8-2$ $\mathrm{cm}$. altae, 1.5-1.8 mm. supra basim diametientes, umbilico lato, ca. $7 \mathrm{~mm}$. diametiente.

Australien: Queensland, Yungaburra, kleiner Baum in ziemlich dünnem Regenwald (C. T. White, im Januar 1918 fruchtend).-Lake Barrine, Atherton Tableland, im Regenwald am Seeufer, sich über das Wasser neigend, 800 m. ü. M., Baum von mittlerer Höhe, häufig ( $S . F$. Kajewski nr. 1135, am 18 Juli 1929 blühend; Typus der Art; Blätter dunkelgrün, Blütenstandsachsen gelbgrün, Petalen weiss).- Range Road, Atherton Tableland, Regenwald, 250 m. ü. M., häufig ( $S . F$. Kajewski nr. 1336, am 7 November blühend und mit unreifen Früchten; mittelhoher Baum bis zu $15 \mathrm{~m}$. hoch, Blütenstandsachsen mit braunen Haaren bekleidet, Sepalen gelbgrün).

Diese Art wurde von C. T. White in Contrib. Arnold Arb. 14: 40. 1933 als Schizomeria floribunda Schlechter angegeben, mit der sie aber nicht vereinigt werden kann, wenn sie mit ihr auch in der Behaarung der Blütenstandachsen etwas Ähnlichkeit hat. Aber Sch. floribunda hat kleinere, stärker kerbig-gesägte, spitze Blätter und um die Hälfte kleinere Blüten.

Botanisches Museum,

BerLin-DahLem. 


\section{$2 \mathrm{BHL}$ Biodiversity Heritage Library}

Mattfeld, Joh. 1939. "Einige Neue Cunoniaceen aus Neuguinea." Journal of the Arnold Arboretum 20(4), 432-436. https://doi.org/10.5962/p.325790.

View This Item Online: https://www.biodiversitylibrary.org/item/33596

DOI: https://doi.org/10.5962/p.325790

Permalink: https://www.biodiversitylibrary.org/partpdf/325790

\section{Holding Institution}

Missouri Botanical Garden, Peter H. Raven Library

\section{Sponsored by}

Missouri Botanical Garden

\section{Copyright \& Reuse}

Copyright Status: In copyright. Digitized with the permission of the rights holder.

Rights Holder: Arnold Arboretum of Harvard University

License: http://creativecommons.org/licenses/by-nc-sa/3.0/

Rights: https://biodiversitylibrary.org/permissions

This document was created from content at the Biodiversity Heritage Library, the world's largest open access digital library for biodiversity literature and archives. Visit BHL at https://www.biodiversitylibrary.org. 